\title{
Simulação em educação médica: uma mudança necessária
}

\author{
Simulation in medical education: a necessary change
}

\author{
Simulación en la educación médica: un cambio necessário
}

\author{
Renato A. Luna," Donn Spight
}

\begin{abstract}
Resumo
Introdução: a educação médica vem sofrendo uma mudança de paradigma devido a uma nova ordem social que se impõe. Esta mudança coloca a simulação como uma ferramenta eficaz para fazer frente aos novos desafios educacionais e sociais do nosso tempo. Este artigo se propõe a introduzir as formas de simulação em educação médica, com ênfase no ensino de cirurgia, bem como contextualizar a mudança de paradigma de ensino e expor alguns resultados do treinamento simulado na prática médica. Materiais e métodos: uma revisão qualitativa da literatura buscando-se artigos que embasam a utilização da simulação como instrumento de educação médica, sobretudo no campo da cirurgia, suas formas e seus impactos na prática clínica foi realizada e seus resultados sumarizados. Resultados: São relatados os fundamentos bem como os objetivos da utilização da simulação, sobretudo na prática cirúrgica. É oferecida uma classificação dos simuladores e exemplos dos resultados de sua utilização na prática clínica. Conclusão: a mudança de paradigma na educacional e na sociedade, bem como os resultados da utilização da simulação na prática clínica a colocam como importante instrumento prático e pedagógico no ensino médico.
\end{abstract}

Descritores: Simulação; Simuladores; Ensino médico.

\begin{abstract}
Introduction: a shift in the medical educational paradigm has been afoot due to a new imposing social order. This social change makes simulation an effective tool for facing the new educational and societal challenges of our time. This review aims to introduce the available models of simulation in medical education, to define the context of this teaching paradigm change and report some results of simulated training in clinical practice. Materials and methods: a qualitative review of the literature based on articles that support the use of simulation as medical education tool, particularly in the field of surgery, its methods and its impact on clinical practice was conducted and results summarized. Results: the foundations and the objectives of the use of simulation, especially in surgical practice, are reported. A classification of simulators and examples of its clinical impact is offered. Conclusion: the paradigm shift in medical education stimulated by a new social order as well as the clinical impact of simulation in daily practices pose simulated training as an important practical and educational tool in medical education.
\end{abstract}

Keywords: Simulation; Simulators; Medical education.

\section{Resumen}

Introducción: la educación médica ha sido objeto de un cambio de paradigma, debido al nuevo orden social que se impone. Este cambio presenta a la simulación como herramienta eficaz para hacer frente a los nuevos retos educativos y sociales de nuestra época. Este artículo pretende introducir las formas de simulación en la educación médica, con énfasis en la enseñanza de cirugía, así como contextualizar el cambio de paradigma en la enseñanza y exponer algunos resultados del entrenamiento simulado en la práctica médica. Materiales y métodos: una revisión cualitativa de la literatura en busca de artículos basados en el uso de la simulación como herramienta de la educación médica, particularmente en el campo de la cirugía, sus formas y su impacto en la práctica clínica se verificó y sus resultados se resumieron. Resultados: los fundamentos así como los objetivos del uso de la simulación son 
descritos, sobre todo en la práctica quirúrgica. Se ofrece una clasificación de los simuladores y ejemplos de los resultados de su uso en la práctica clínica. Conclusión: el cambio de paradigma en la educación y en la sociedad, así como los resultados del uso de la simulación en la práctica clínica, la presentan como herramienta práctica y pedagógica en la enseñanza médica.

Palabras clave: Simulación; Simuladores; Enseñanza médica.

\section{Introdução}

A utilização da simulação na educação médica é uma realidade nos principais centros universitários do mundo. Ela é um instrumento importante na transição de um modelo educacional centrado no professor, alicerçado na relação mestre-aprendiz, para um modelo centrado no aluno, baseado nas interações multidisciplinares mais próximas da realidade profissional diária e mais preocupado com as necessidades dos pacientes.

A crescente demanda da sociedade por uma maior segurança nas práticas clínicas e o aumento do custo médico criam o pano de fundo ideal para a implementação de programas de simulação. Neles, o desenvolvimento de uma habilidade não depende da chegada aleatória de pacientes para o treinamento e a realização de procedimentos, podendo ocorrer em ambiente controlado com a presença de tutores experimentados e com consequências mínimas em caso de falhas, evitando a exposição dos pacientes. Com o treinamento simulado, espera-se um aprendizado mais homogêneo entre os estudantes, menor dependência do aparecimento randômico de casos e o aumento da segurança para os pacientes submetidos aos procedimentos treinados.

Neste artigo, abordaremos as bases e razões para a adoção do treinamento simulado nos currículos médicos das universidades.

\section{Fundamentos para utilização da simulação}

O estudante de medicina se depara com inúmeros desafios durante seu treinamento. Inicialmente, ele aprende o vocabulário básico da profissão, assim como a fisiologia e fisiopatologia das enfermidades mais prevalentes. Sinais e sintomas são descritos; porém, na prática diária é necessário que o médico extraia e interprete os achados clínicos para efetuar um diagnóstico e um tratamento corretos, ficando clara a existência de uma lacuna entre o ensinado e o necessário para a prática profissional diária.

Em uma segunda fase, o estudante se prepara para a tradução da teoria para a prática, iniciando o aprendizado em serviço, ou o internato. Devido às crescentes pressões sociais para eficiência e à menor tolerância ao aprendizado em pacientes, menos oportunidades são oferecidas aos estudantes. Some-se a isso fato de o processo de seleção para a próxima fase da carreira no Brasil, a residência médica, ser baseado em prova teórica simples. Em consequência, o que se observa é um esvaziamento desta fase acadêmica, gerando residentes cada vez menos preparados para a prática clínica rotineira.

$\mathrm{Na}$ terceira fase, a residência propriamente dita, a tônica é o aprendizado em serviço com foco em uma especialidade. Este momento é baseado no modelo halstediano, no qual um aprendiz é tutorado por um mestre mais experiente. Aqui o aprendizado depende diretamente da exposição de cada estudante a cada caso e do volume de casos atendidos em um determinado período, oferecendo um conhecimento heterogêneo que depende da experiência de cada trainee. A crescente tendência à diminuição do número e da duração das internações é um obstáculo adicional a este modelo.'

Após a residência, as oportunidades de reciclagem, atualização e aprendizado de novas técnicas são limitadas, tendo que ser conciliadas com as atividades profissionais diárias.

Tudo isto somado a um ambiente médico legal crescentemente inóspito cria um ambiente ideal para o desenvolvimento de atividades de simulação em ambiente protegido, em que um conhecimento uniforme pode ser transmitido aos estudantes, que podem ser treinados em diversos cenários clínicos preestabelecidos. 


\section{Definição e objetivos da simulação médica}

Simulação pode ser definida como a técnica utilizada para substituir ou amplificar experiências reais, geralmente em ambiente de imersão total, que evoca ou replica aspectos essenciais da prática diária, de uma forma interativa. ${ }^{2} \mathrm{O}$ treinamento em simulação tem como objetivo maior melhorar a qualidade e a segurança do atendimento oferecido aos pacientes. Para atingir estes objetivos é necessário estabelecer metas a serem atingidas e um método de treinamento validado para atingir estas metas. Independentemente da forma do currículo estabelecida, é de fundamental importância a definição dos padrões técnicos e dos objetivos didáticos da atividade simulada. Este currículo deve permitir prática por livre demanda, complexidade progressiva das tarefas e oferecer constante feedback ao aluno.

A prática livre se refere à prática repetitiva e focada de uma tarefa de forma supervisionada, permitindo assim a correção imediata de eventuais erros cometidos. Em outras palavras, são necessários acesso livre ao simulador e feedback de um tutor sobre o desempenho do aluno. ${ }^{3}$

Em geral, os currículos podem se basear em tempo de treinamento/número de repetições da atividade ou em metas de proficiência a serem atingidas. Os currículos que buscam proficiência oferecem maior flexibilidade e eficiência na utilização dos recursos de treinamento e garantem que todos os trainees atinjam um determinado nível de aptidão ao final do treinamento. Também a implementação de um currículo progressivo, no qual a dificuldade da tarefa aumenta com a progressão do treinamento, é importante para garantir um melhor aprendizado por parte do trainee. $^{4}$

A importância do feedback no processo de aprendizado já está bem demonstrada na simulação médica, uma vez que ele muito facilita a compreensão do trainee no processo de aprendizado. ${ }^{5}$ No entanto, a intensidade do feedback necessário para o melhor aprendizado ainda não é claramente compreendida. Em um trabalho bem planejado, grupos foram determinados de acordo com a intensidade do feedback oferecido. O primeiro grupo teve retorno intenso e frequente durante
$100 \%$ do tempo de treinamento. O segundo grupo recebeu feedback limitado a 10 minutos por hora de sessão. Um terceiro grupo teve o feedback restrito a 10 minutos por hora de treinamento e acesso a um vídeo de orientação disponível durante o treinamento. Como resultado, os grupos com feedback limitado tiveram uma curva de aprendizado mais rápida do que o grupo com feedback intensivo. ${ }^{6}$ Acredita-se que a experiência ganha para se superar as dificuldades das tarefas de forma independente traz mais benefícios que uma correção intensiva e imediata, que pode evitar que o aprendiz reconheça os elementos fundamentais para a realização da atividade com sucesso.

\section{Classificação dos simuladores}

Diferentes tipos de simuladores estão disponíveis e são utilizados no meio médico. Eles podem ser divididos em: modelos de bancada, modelos animais, cadáveres humanos, simuladores de performance humana e simuladores de realidade virtual. $^{7}$

Os modelos de bancada são modelos inanimados, geralmente de baixo custo, portáteis e reutilizáveis. Sua principal vantagem é a possibilidade de fragmentação de tarefas complexas em seus elementos essenciais. Funcionam muito bem para o ensino de tarefas básicas para iniciantes. Sua limitação principal é a baixa fidelidade oferecida, o que restringe sua capacidade de simular tarefas complexas.

Modelos de simulação em animais vivos oferecem alta fidelidade na simulação de tarefas complexas, como cirurgias em condições "reais". São úteis porque reproduzem o comportamento do tecido vivo e testam habilidades técnicas avançadas que influenciam o resultado final de uma cirurgia, como sutura laparoscópica intracorpórea, hemostasia, anastomoses etc. O alto custo, necessidade de pessoal e instalações especializadas, considerações éticas, diferenças anatômicas e impossibilidade de reutilização limitam o uso disseminado desta técnica.

A utilização de cadáveres oferece a maior fidelidade dentre os modelos de simulação e permitem a reprodução de procedimentos de alta complexidade. Eles são melhores utilizados para treinamento de procedimentos específicos $\mathrm{e}$ habilidades de dissecção. O custo, disponibilidade, 
riscos de infecção e considerações éticas limitam sua aplicação.

Simuladores de performance humana podem ser usados repetidamente com alta fidelidade e interatividade. Manequins com diferentes níveis de sofisticação podem ser usados em diversos cenários, incluindo situações multidisciplinares e interprofissionais para treinamento de equipes e gerenciamento de crises. As principais limitações são os custos e a limitação na aplicação de procedimentos, valendo-se mais para definir um padrão de resposta organizado em vez de uma habilidade técnica específica.

A simulação virtual tem como vantagens ser reutilizável e poder capturar e armazenar dados de desempenho, permitindo um feedback ao aluno e o monitoramento da progressão do treinamento. Ela pode ser utilizada tanto para aquisição de habilidades básicas como avançadas, sendo amplamente aplicada no treinamento de procedimentos endoscópicos e laparoscópicos. Suas principais limitações são custo, manutenção e ausência de sensação tátil.

\section{Resultados do treinamento}

\section{simulado}

Após essa discussão inicial sobre simulação médica, a questão que se impõe é o resultado deste treinamento na prática clínica. Vários exemplos de transferência de habilidades do laboratório para a prática diária estão disponíveis na literatura. Residentes de cirurgia que treinaram até atingir marcos de proficiência em um modelo de fechamento de fáscia apresentaram melhor performance na sala de cirurgia quando comparados a residentes não treinados. $\mathrm{O}$ instrumento de avaliação aplicado foi o Objective Structured Assessment of Technical Skills (OSATS). ${ }^{8}$ Residentes treinados neste simulador apresentaram até mesmo melhor performance cognitiva do que os não treinados, sugerindo que a aquisição da habilidade básica em laboratório permite ao trainee focar de maneira mais efetiva em outros aspectos cognitivos envolvidos em uma cirurgia real. ${ }^{9} \mathrm{Um}$ dado interessante é que alunos treinados que atingem a automação da tarefa demonstram melhor transferência de habilidade ao cenário real do que aqueles treinados para atingir a proficiência..$^{10}$
Em outro exemplo, residentes treinados para inserção de cateteres centrais por acesso periférico (PICC lines) em simuladores se saíram melhor na tarefa do que residentes treinados pelo método habitual baseado no aprendizado em serviço, demonstrando transferência de habilidades do laboratório para a prática clínica." Outros trabalhos com nível I de evidência corroboram estes exemplos em diferentes especialidades, desde procedimentos colonoscópicos até urológicos. ${ }^{12,13}$

É importante salientar que a aquisição de conhecimento não se restringe a habilidades manuais. Estudantes treinados em simuladores para identificar sopro sistólico aórtico se saíram melhor que seus pares treinados de forma tradicional. ${ }^{14}$ Uma análise qualitativa da literatura identificou benefícios do treinamento simulado na saúde pública. ${ }^{15} \mathrm{Em}$ um hospital terciário de 900 leitos foi demonstrado em uma análise dos custos de um treinamento simulado para inserção de cateter venoso central que a redução dos níveis de infecções relacionadas aos cateteres gerou uma economia de 700 mil dólares, enquanto os custos de treinamento foram estimados em torno de 112 mil dólares. Ou seja, houve uma proporção de 7 para 1 em favor do treinamento simulado. ${ }^{16}$

\section{Conclusão}

Está clara a mudança do modelo de educação, migrando da estrutura baseada no professor e na relação mestre-aprendiz para um modelo, centrado no aluno, que incorpora os princípios da multidisciplinaridade. Diversos estudos demonstram que, quando aplicado corretamente, um currículo de simulação leva a uma melhora das habilidades clínicas, do desempenho na sala de cirurgia, da comunicação interna da equipe e de sua eficiência como grupo. Quando consideramos que o tempo de treinamento do médico se mantém o mesmo apesar do substancial aumento de conhecimento e tecnologia na medicina no último século, fica claro que o paradigma tradicional de treinamento não é mais adequado. Limitações da carga horária de treinamento associadas às demandas médico-legais e financeiras e às pressões sociais por maior segurança dos pacientes formam o pano de fundo perfeito para a adoção das técnicas de simulação na educação médica. Já estamos atrasados... 


\section{Referências}

1. Roos NP1, Shapiro E. Using the information system to assess change: the impact of downsizing the acute sector. Med Care. 1995;33(12 Suppl):DS109-26.

2. Gaba DM. The future vision of simulation in health care. Qual Saf Health Care. 2004;13 Suppl 1:i2-10.

3. Ericsson KA. Deliberate practice and the acquisition and maintenance of expert performance in medicine and related domains. Acad Med. 2004;79(10 Suppl):S70-81.

4. Ali MR, Mowery Y, Kaplan B, DeMaria EJ. Training the novice in laparoscopy. More challenge is better. Surg Endosc. 2002;16(12):1732-6.

5. Xeroulis GJ, Park J, Moulton CA, Reznick RK, Leblanc $\mathrm{V}$, Dubrowski A. Teaching suturing and knot-tying skills to medical students: a randomized controlled study comparing computer-based video instruction and (concurrent and summary) expert feedback. Surgery. 2007;141(4):442-9.

6. Stefanidis D, Korndorffer JR Jr, Heniford BT, Scott DJ. Limited feedback and video tutorials optimize learning and resource utilization during laparoscopic simulator training. Surgery. 2007;142(2):202-6.

7. Reznick RK, MacRae H. Teaching surgical skills--changes in the wind. N Engl J Med. 2006;355(25):2664-9.

8. Martin JA, Regehr G, Reznick R, MacRae H, Murnaghan J, Hutchison C, et al. Objective structured assessment of technical skill (OSATS) for surgical residents. Br J Surg. 1997;84(2):273-8.

9. Palter VN, Grantcharov T, Harvey A, Macrae HM. Ex vivo technical skills training transfers to the operating room and enhances cognitive learning: a randomized controlled trial. Ann Surg. 2011;253(5):886-9. http://dx.doi.org/10.1097/SLA.0b013e31821263ec

10. Stefanidis D, Scerbo MW, Montero PN, Acker CE, Smith WD. Simulator training to automaticity leads to improved skill transfer compared with traditional proficiency-based training: a randomized controlled trial. Ann Surg. 2012;255(1):30-7. http://dx.doi. org/10.1097/SLA.0b013e318220ef31
11. Andreatta P, Chen Y, Marsh M, Cho K. Simulation-based training improves applied clinical placement of ultrasound-guided PICCs. Support Care Cancer. 2011;19(4):539-43. http://dx.doi.org/10.1007/ s00520-010-0849-2

12. Haycock A, Koch AD, Familiari P, van Delft F, Dekker E, Petruzziello L, et al. Training and transfer of colonoscopy skills: a multinational, randomized, blinded, controlled trial of simulator versus bedside training. Gastrointest Endosc. 2010;71(2):298-307. http://dx. doi.org/10.1016/j.gie.2009.07.017

13. Schout BM, Ananias HJ, Bemelmans BL, d'Ancona FC, Muijtjens AM, Dolmans VE, et al. Transfer of cysto-urethroscopy skills from a virtual-reality simulator to the operating room: a randomized controlled trial. BJU Int. 2010;106(2):226-31; discussion 231. http://dx.doi.org/10.1111/j.1464-410X.2009.09049.x

14. Fraser K, Wright B, Girard L, Tworek J, Paget M, Welikovich L, et al. Simulation training improves diagnostic performance on a real patient with similar clinical findings. Chest. 2011;139(2):376-81. http:// dx.doi.org/10.1378/chest.10-1107

15. McGaghie WC, Draycott TJ, Dunn WF, Lopez CM, Stefanidis D. Evaluating the impact of simulation on translational patient outcomes. Simul Healthc. 2011;6 Suppl:S42-7. http://dx.doi.org/10.1097/SIH.0b013e318222 fde 9

16. Cohen ER, Feinglass J, Barsuk JH, Barnard C, O'Donnell A, McGaghie WC, et al. Cost savings from reduced catheter-related bloodstream infection after simulation-based education for residents in a medical intensive care unit. Simul Healthc. 2010;5(2):98102. http://dx.doi.org/10.1097/SIH.0b013e3181bc8304

\section{Renato A. Luna}

Serviço de cirurgia geral, Hospital dos Servidores do Estado do Rio de Janeiro. Rio de Janeiro, RJ, Brasil.

\section{Donn Spight}

Surgery simulation center, Oregon Health \& Science University. Portland, OR, United States. 